$\mathbb{T}$ periodica polytechnica

Civil Engineering

$54 / 2(2010) 89 \sqrt{94}$

doi: 10.3311/pp.ci.2010-2.03

web: http://www.pp.bme.hu/ci

(c) Periodica Polytechnica 2010

RESEARCH ARTICLE

\section{Spatial and temporal separation in overdamped systems}

\author{
András Bibó / György Károlyi
}

Received 2010-05-26, accepted 2010-06-23

\begin{abstract}
Due to their small sizes molecular systems are often overdamped. Conformational changes in these molecules are a consequence of the separation of the energy input between the different displacements and velocities of the different functional sites of the molecule. We show how a simple mechanical device, that splits the forces between the different parts of the system, can achieve spatial (displacements) and temporal (velocities) separation. As a result of the overdamped nature of the motion, the equations can be decoupled by introducing a damping center. As a particular example, we briefly discuss myosin II, a motor protein responsible for the contraction of skeletal muscles.
\end{abstract}

\section{Keywords}

Overdamped dynamics $\cdot$ multiple spatial scales $\cdot$ multiple time scales $\cdot$ myosin II

\section{Acknowledgement}

Financial support from OTKA under grant no. K 68415 and IN 82349 are hereby gratefully acknowledged. We are indebted for the valuable discussions with Mihály Kovács.

\section{András Bibó}

Department of Structural Mechanics, BME, H-1111 Budapest, Múegyetem rkp. 3., Hungary

e-mail: biboan@gmail.com

\section{György Károlyi}

Department of Structural Mechanics, BME, H-1111 Budapest, Múegyetem rkp. 3., Hungary

e-mail: karolyi@tas.me.bme.hu

\section{Introduction}

In engineering systems, damping is usually present [1].2], and plays an important role. In cases, damping can be so strong that the system is overdamped [3-6]. For example, if we assume a single material point of mass $m$ fixed by a linear spring of strength $k$ driven by a constant force $F_{0}$, in the presence of damping, we can write Newton's second law in the form

$$
F_{0}-c \dot{y}-k y=m \ddot{y},
$$

where $y=y(t)$ is the displacement of the mass, and dots indicate derivation with respect to time $t$. This system is overdamped if the damping coefficient $c>2 \sqrt{\mathrm{km}}$, in that case the mass does not start oscillating, it just relaxes towards its equilibrium state [6].

Overdamped systems are not unique for engineering structures. High level of damping is found when motion occurs in fluids of high viscosity, like in case of colloidal particles in magnetorheological suspensions [7.-9] or motion of flagellar organisms [10,11]. Overdamped coupled pendulums are also used to model Josephson junctions [12,13]. In many cases, the small size and mass of the particles is the reason why the system is overdamped: damping is proportional to the second, while mass to the third power of the characteristic size of the particles, for further examples see Refs. [14-16]. In some cases, damping can be so high that it dominates the inertial term in Eq. (1). Indeed, we can cast (1) into a dimensionless form to be able to compare the importance of the terms. Measuring time in units of $\tau=c / k$, displacements in units of $L=F_{0} / k$, so that the new, dimensionless time variable is $t^{\prime}=t / \tau$ and the new, dimensionless displacement is $x=y / L$, Eq. (1) can be written in the form

$$
\frac{m k}{c^{2}} \ddot{x}+\dot{x}+x=1,
$$

where dot indicates derivation with respect to dimensionless time $t^{\prime}$. From this form we can see that if $m k / c^{2}$ is much less than one, the inertial term $m k \ddot{x} / c^{2}$ can be neglected. In this paper we intend to investigate such systems where damping is extremely strong. In this case, the system can be described by first order equations of motion: the damping term, containing 
the velocity, is equal to the other forces acting on the mass: the spring force and the load $F_{0}$.

We note that mathematically, when the coefficient of the leading order term in (2) is very small, the system is singularly perturbed. This means that the first term, containing the second order derivatives, only plays a role when the other terms vanish or much smaller. A general treatment of such systems usually requires special methods, like multiple-scale analysis or matched asymptotic expansion [17]. However, our overdamped system can be treated as first order: in our analysis we only take a look at the decaying motion of the system where the order of magnitude of the accelerations is never larger than that of the velocities.

The motivation for investigating such very strongly damped systems comes from the observation that molecular systems in living organisms possess such properties. For example, in case of the motor protein called myosin II, an enzyme responsible for contraction of skeletal muscles [18], measurements serve the following data: the mass of the molecule is $m=160 \times 10^{-24} \mathrm{~kg}$ [19], the restoring molecular forces impose a spring constant on the order of $k=4 \mathrm{pN} / \mathrm{nm}$ [19], while damping is $c=60 \mathrm{pN} \cdot \mathrm{s} / \mathrm{m}$ [19]. For determining the type of motion, we need to compare the inertial term with the damping term by calculating the value of $m k / c^{2}$. In case of myosin II, we find $m k / c^{2}=1.78 \times 10^{-4} \ll$ 1 which indicates that the motion of myosin II molecules during muscle contraction is highly overdamped, and the inertial term can be neglected safely from (1).

Even a single molecule can have several functional sites, with displacements that can strongly differ from each other. The displacements are caused by a chemical energy input, transformed to mechanical energy. In living organisms, there are special proteins called motor proteins that are capable of performing the transformation at a high rate of efficiency. The spatial structure of these molecules is called conformation which can completely change during the working cycle of the protein. In the case of myosin II, for example, after the hydrolysis of adenosine triphosphate (ATP) the energy gain results in a conformational change at two parts of myosin II: there is a rotational displacement at a part of the molecule called converter domain, and another motion at a place called actin binding site which makes the molecule tend to attach to a filamentous protein chain called actin [18]. To achieve a conformational change, the energy input has to lead to a different displacement of the different parts of the molecule. Hence it is of interest to see, through a simple mechanical model, how different displacements can be achieved in a simple overdamped system under the action of a single force. This is the subject of Sec. 2. how a simple mechanical device can lead to spatial separation, that is, to different displacements of the parts of a simple mechanical system. We note that these different displacements can be tuned to fit any prescribed values by an appropriate choice of the system parameters.

In many cases, however, it is not only the final position of the parts of the molecules, at the end of a conformational change, that is of importance. For instance, the speed of the approach of the actin filament by a myosin II molecule can be much faster than the motion of its loaded lever arm. To address this question, in Sec. 3 we investigate an extended version of the model that is capable to provide different speeds while approaching a final state under the action of a single force. This leads to temporal separation, that is, the time required to reach the prescribed final displacaments can differ strongly for each body of the system. Hence in this system there is both spatial and temporal separation. Finally, in Sec. 4 we draw our conclusions.

\section{Separation of displacements}

The system we investigate in this section is shown in Fig. 19. The two bodies of masses $m_{1}$ and $m_{2}$ are connected to point $\mathrm{P}$ by linear springs of stiffness $k_{1}$ and $k_{2}$, respectively. This point $\mathrm{P}$ is driven by a force $F$ that might depend on time: $F(t)$. The displacement of point mass $m_{1}$ is $y_{1}$, that of $m_{2}$ is $y_{2}$. The displacement of point $\mathrm{P}$ is $y$. The motion of the masses is damped, the damping coefficients are $c_{1}$ and $c_{2}$ for the two masses, respectively.

The forces in the springs are

$$
S_{1}=\left(y_{1}-y\right) k_{1}, \quad S_{2}=\left(y-y_{2}\right) k_{2}
$$

The forces exerted on the masses are from these spring forces and from damping:

$$
-S_{1}-c_{1} \dot{y}_{1}=m_{1} \ddot{y}_{1}, \quad S_{2}-c_{2} \dot{y}_{2}=m_{2} \ddot{y}_{2},
$$

where dots indicate derivation with respect to time. For simplicity, we assume that point $\mathrm{P}$ is massless and is not affected by damping, that is, it serves only as a force splitting device. Hence the forces have to balance each other:

$$
S_{1}+F=S_{2}
$$

This implies the relation $\left(y_{1}-y\right) k_{1}+F=\left(y-y_{2}\right) k_{2}$, leading to

$$
y=\frac{F+k_{1} y_{1}+k_{2} y_{2}}{k_{1}+k_{2}} .
$$

Substituting this and the spring forces into Eqs. (4) gives

$$
\begin{aligned}
& m_{1} \ddot{y}_{1}=-c_{1} \dot{y}_{1}+F \frac{k_{1}}{k_{1}+k_{2}}-\frac{k_{1} k_{2}}{k_{1}+k_{2}}\left(y_{1}-y_{2}\right) \\
& m_{2} \ddot{y}_{2}=-c_{2} \dot{y}_{2}+F \frac{k_{2}}{k_{1}+k_{2}}+\frac{k_{1} k_{2}}{k_{1}+k_{2}}\left(y_{1}-y_{2}\right)
\end{aligned}
$$

We can cast this system of equations in a dimensionless form. Let's write force $F$ in the form $F(t)=F_{0} f(t)$ where $F_{0}$ is the magnitude of the force and $|f(t)| \leq 1$ the dimensionless force function that describes the time-dependence of the force. Then we can measure the displacements in terms of $L=\left(k_{1}+\right.$ $\left.k_{2}\right) F_{0} / k_{1} k_{2}$, the time in terms of $\tau=c_{1} c_{2}\left(k_{1}+k_{2}\right) /\left[k_{1} k_{2}\left(c_{1}+\right.\right.$ $c_{2}$ )], so that Eqs. (7) become

$$
\begin{aligned}
\mu_{1} \ddot{x}_{1} & =-\gamma_{1} \dot{x}_{1}+\kappa_{1} f(t)-x_{1}+x_{2}, \\
\mu_{2} \ddot{x}_{2} & =-\gamma_{2} \dot{x}_{2}+\kappa_{2} f(t)+x_{1}-x_{2},
\end{aligned}
$$




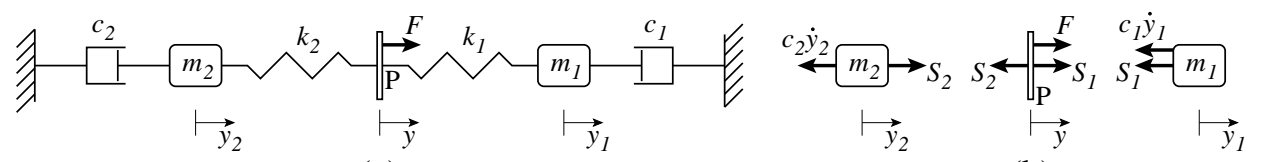

(a)

(b)

Fig. 1. (a) Layout of the overdamped system. (b) Free body diagrams showing the forces acting on each pieces.

where $x_{i}=y_{i} / L, i=1,2$, dots indicate derivation with respect to dimensionless time $t / \tau, \mu_{1}=m_{1} L / F_{0} \tau^{2}$ and $\mu_{2}=$ $m_{2} L / F_{0} \tau^{2}$ are the dimensionless masses, $\gamma_{1}=\left(c_{1}+c_{2}\right) / c_{2}$ and $\gamma_{2}=\left(c_{1}+c_{2}\right) / c_{1}$, with $\gamma_{1}^{-1}+\gamma_{2}^{-1}=1$, characterize damping of the two bodies, and $\kappa_{1}=k_{1} /\left(k_{1}+k_{2}\right)$ and $\kappa_{2}=k_{2} /\left(k_{1}+k_{2}\right)$ are the relative spring stiffnesses with $\kappa_{1}+\kappa_{2}=1$.

If the system is overdamped in the sense described in the Introduction, then $\mu_{1}$ and $\mu_{2}$ are negligible. This leads to the system of equations

$$
\gamma_{1} \dot{x}_{1}=\kappa_{1} f(t)-x_{1}+x_{2}, \quad \gamma_{2} \dot{x}_{2}=\kappa_{2} f(t)+x_{1}-x_{2} .
$$

To solve this set of equations, we introduce the concept of the damping center, so that its position is defined as $c(t)=$ $\gamma_{1} x_{1}(t)+\gamma_{2} x_{2}(t)$. In an overdamped system, the damping center is similar to the center of mass in inertial systems: we can solve the system by using the location $c(t)$ of the damping center as one of the unknowns and the increase $d(t)=x_{1}(t)-x_{2}(t)$ in the distance between the bodies as the other unknown. This way Eqs. (9) can be cast into the following form:

$$
\dot{c}=f(t), \quad \dot{d}=\left(\frac{\kappa_{1}}{\gamma_{1}}-\frac{\kappa_{2}}{\gamma_{2}}\right) f(t)-d,
$$

where we have used the fact that $\kappa_{1}+\kappa_{2}=1$. The advantage of this form is that the equations are now de-coupled, there is now only a single unknown in each equation. Once these equations are solved, we find $x_{1}$ and $x_{2}$ from

$$
x_{1}(t)=\frac{c(t)+\gamma_{2} d(t)}{\gamma_{1}+\gamma_{2}}, \quad x_{2}(t)=\frac{c(t)-\gamma_{1} d(t)}{\gamma_{1}+\gamma_{2}} .
$$

We solve Eqs. 10 in the special case of a constant driving force $f(t) \equiv 1$. In this case we find

$$
c(t)=t+A, \quad d(t)=\left(\frac{\kappa_{1}}{\gamma_{1}}-\frac{\kappa_{2}}{\gamma_{2}}\right)+B e^{-t},
$$

where $A$ and $B$ can be set from the initial conditions. If at the start of a conformational change, at $t=0$ the system is at rest, $c(0)=0$ and $d(0)=0$, we find $A=0$ and $B=\kappa_{2} / \gamma_{2}-\kappa_{1} / \gamma_{1}$, and hence

$$
\begin{gathered}
c(t)=t, \\
d(t)=\left(\frac{\kappa_{1}}{\gamma_{1}}-\frac{\kappa_{2}}{\gamma_{2}}\right)\left(1-e^{-t}\right) .
\end{gathered}
$$

This means that the damping center will move at a constant speed 1. The distance of the two mass, after a short transient, converges to $\kappa_{1} / \gamma_{1}-\kappa_{2} / \gamma_{2}$. The convergence is exponential, with rate 1 .
In the original dimensionless variables the result is

$$
\begin{aligned}
& x_{1}(t)=\frac{1}{\gamma_{1}+\gamma_{2}}\left[t+\left(\frac{\gamma_{2}}{\gamma_{1}} \kappa_{1}-\kappa_{2}\right)\left(1-e^{-t}\right)\right], \\
& x_{2}(t)=\frac{1}{\gamma_{1}+\gamma_{2}}\left[t-\left(\kappa_{1}-\frac{\gamma_{1}}{\gamma_{2}} \kappa_{2}\right)\left(1-e^{-t}\right)\right] .
\end{aligned}
$$

The first term in the square bracket gives a constant velocity translation, which is the same for both masses. The second term contains an exponential term that decays rapidly, and a constant term that gives the long time displacement of the bodies. More precisely, we can compute $x_{1}-x$ and $x-x_{2}$ as the distance of the masses from point $\mathrm{P}$, where $x=y / L$ is the dimensionless position of point P. Using (6) we find that $x=\kappa_{1} \kappa_{2}+\kappa_{1} x_{1}+$ $\kappa_{2} x_{2}$, which leads to $x_{1}-x=-\kappa_{1} \kappa_{2}+\kappa_{2}\left(x_{1}-x_{2}\right)$ and $x-x_{2}=$ $\kappa_{1} \kappa_{2}+\kappa_{1}\left(x_{1}-x_{2}\right)$. Using (14), in the long time limit $t \rightarrow \infty$ we find

$$
\begin{aligned}
& x_{1}-x=-\kappa_{1} \kappa_{2}+\kappa_{2}\left(\frac{\kappa_{1}}{\gamma_{1}}-\frac{\kappa_{2}}{\gamma_{2}}\right), \\
& x-x_{2}=\kappa_{1} \kappa_{2}+\kappa_{1}\left(\frac{\kappa_{1}}{\gamma_{1}}-\frac{\kappa_{2}}{\gamma_{2}}\right) .
\end{aligned}
$$

Hence the displacements of the bodies with respect to point $\mathrm{P}$, driven by the force, are different, hence their positions are spatially separated. This spatial separation depends on the stiffnesses of the springs and on the relative strength of damping. This is a very important finding in terms of the conformational changes in molecules: after a conformational change different functional units of the molecules can end up in separate final states using a similar mechanism.

Note, however, that the velocities cannot be separated this way, the long-time velocity is the same for the two bodies, and it coincides with the velocity of point $\mathrm{P}$. In the next section we modify this model to find a mechanical device that, besides different displacements, can also lead to different velocities of its parts, and hence these parts can approach their different final states during different time periods.

\section{Separation of velocities}

Simple mechanical devices that can, as we shall see, separate the velocities as well as the displacements are shown in Figs. 2 and 3 In case of the device shown in Fig. 2 a the two ratcheted bars are moved by the rotating cylinders, that, in turn, is rotated by the force $F$ acting on the third ratcheted bar. As the radius of the cylinders are different, $\alpha R$ for the bar attached to mass $m_{1}$ and $\beta R$ for the bar attached to $m_{2}$, and it is $R$ for the force $F$, we can effectively exert a different force on the masses. As before, the displacements of the masses are $y_{1}$ and $y_{2}$, while the displacement of the loaded racheted bar is $y$. 


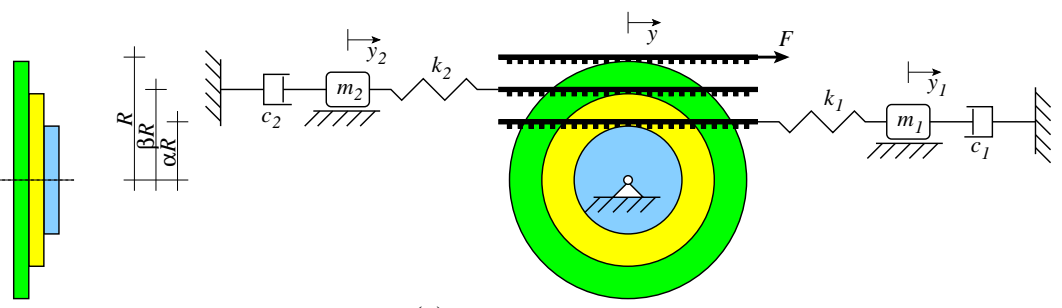

(a)

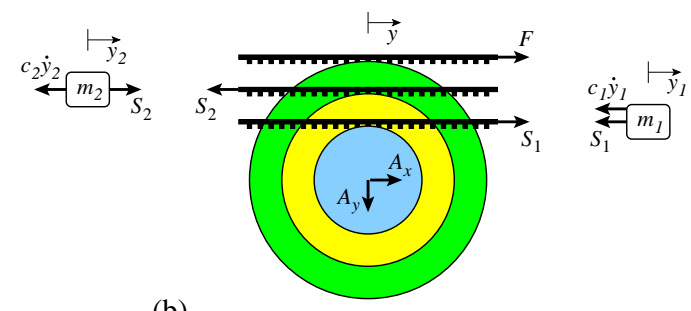

(b)

Fig. 2. (a) Layout of the force splitting device using cylinders. (b) Free body diagrams showing the forces acting on each pieces.

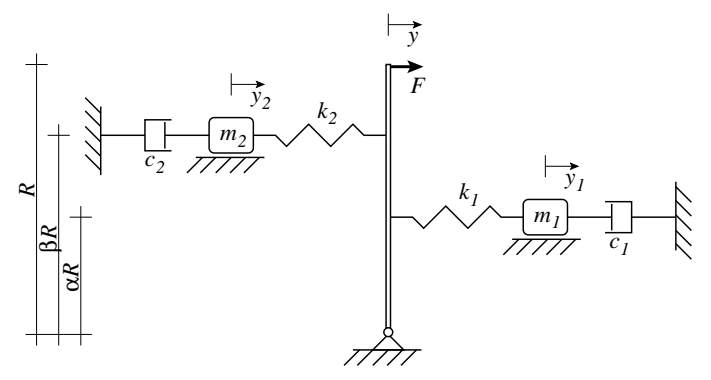

(a)

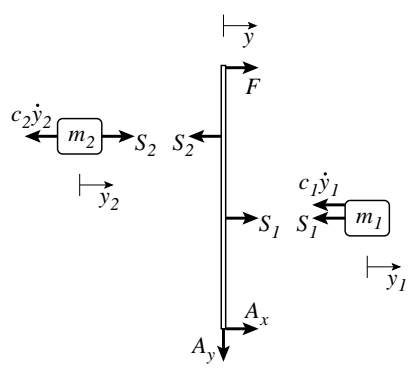

(b)

Fig. 3. (a) Layout of the force splitting device using a lever arm. (b) Free body diagrams showing the forces acting on each pieces.

Fig. 33 shows a device that separates the forces by applying a lever arm. This works effectively the same way as the previous device shown in Fig. 2, but only when the horizontal displacement $y$ of the top of the lever arm is small. If this is the case, the two systems work essentially the same way. The forces acting on the pieces are shown in Figs. $2 \mathrm{~b}$ and $3 \mathrm{~b}$. Assuming neither inertia nor damping for these force separating devices, we can write the balance of moments around the pinned point of both devices as

$$
S_{1} \alpha R+F R=S_{2} \beta R .
$$

Here $S_{1}=\left(y_{1}-\alpha y\right) k_{1}$ and $S_{2}=\left(\beta y-y_{2}\right) k_{2}$ are the forces in the springs, and from we can express $y$ as

$$
y=\frac{F+\alpha k_{1} y_{1}+\beta k_{2} y_{2}}{\alpha^{2} k_{1}+\beta^{2} k_{2}} .
$$

Substituting this into (4) we find the equations of motion for the two masses to be

$$
\begin{gathered}
m_{1} \ddot{y}_{1}=-c_{1} \dot{y}_{1}+\frac{\alpha k_{1} F}{\alpha^{2} k_{1}+\beta^{2} k_{2}}-\frac{\beta^{2} k_{1} k_{2}}{\alpha^{2} k_{1}+\beta^{2} k_{2}} y_{1} \\
+\frac{\alpha \beta k_{1} k_{2}}{\alpha^{2} k_{1}+\beta^{2} k_{2}} y_{2}, \\
m_{2} \ddot{y}_{2}=-c_{2} \dot{y}_{2}+\frac{\beta k_{2} F}{\alpha^{2} k_{1}+\beta^{2} k_{2}}+\frac{\alpha \beta k_{1} k_{2}}{\alpha^{2} k_{1}+\beta^{2} k_{2}} y_{1} \\
-\frac{\alpha^{2} k_{1} k_{2}}{\alpha^{2} k_{1}+\beta^{2} k_{2}} y_{2} .
\end{gathered}
$$

Note that with the choice of $\alpha=\beta=1$ we recover Eq. (7).

Let us, again, cast this system of equations into a dimensionless form. We can measure the distances in units of $L=$ $\left(\alpha^{2} k_{1}+\beta^{2} k_{2}\right) F_{0} /\left(\alpha \beta k_{1} k_{2}\right)$ and time in units of $\tau=\left(\alpha^{2} k_{1}+\right.$ $\left.\beta^{2} k_{2}\right) c_{1} c_{2} /\left(\alpha \beta k_{1} k_{2}\left(c_{1}+c_{2}\right)\right)$, then we obtain the new dimensionless equations of motion:

$$
\begin{aligned}
& \mu_{1} \ddot{x}_{1}=-\gamma_{1} \dot{x}_{1}+\kappa_{1} f(t)-\frac{\beta}{\alpha} x_{1}+x_{2}, \\
& \mu_{2} \ddot{x}_{2}=-\gamma_{2} \dot{x}_{2}+\kappa_{2} f(t)+x_{1}-\frac{\alpha}{\beta} x_{2},
\end{aligned}
$$

where we use the notation $x_{i}=y_{i} / L, i=1,2$, with dots indicating derivation with respect to dimensionless time $t / \tau$. The parameters $\mu_{1}=m_{1} L / F_{0} \tau^{2}$ and $\mu_{2}=m_{2} L / F_{0} \tau^{2}$ indicate the dimensionless masses just like in the previous section, $\gamma_{1}=\left(c_{1}+c_{2}\right) / c_{2}$ and $\gamma_{2}=\left(c_{1}+c_{2}\right) / c_{1}$ characterize damping of the two masses. The parameters that differ from the previous, simpler model are the relative spring stiffnesses $\kappa_{1}=\alpha k_{1} /\left(\alpha^{2} k_{1}+\beta^{2} k_{2}\right)$ and $\kappa_{2}=\beta k_{2} /\left(\alpha^{2} k_{1}+\beta^{2} k_{2}\right)$. Note, however, that with the choice of $\alpha=\beta=1$ the old $\kappa_{1}$ and $\kappa_{2}$ are recovered. Also note that $\alpha \kappa_{1}+\beta \kappa_{2}=1$ holds.

Just like for the model in the previous section, we can introduce the concept of the damping center, whose position is defined as $c(t)=\alpha \gamma_{1} x_{1}(t)+\beta \gamma_{2} x_{2}(t)$. We also introduce the normalized distance between the masses as $d(t)=\beta x_{1}(t)-\alpha x_{2}(t)$. With these notations, Eqs. 19] can be rewritten as

$$
\begin{aligned}
& \dot{c}(t)=f(t), \\
& \dot{d}(t)=\left(\frac{\beta \kappa_{1}}{\gamma_{1}}-\frac{\alpha \kappa_{2}}{\gamma_{2}}\right) f(t)-\frac{\alpha^{2} \gamma_{1}+\beta^{2} \gamma_{2}}{\alpha \beta \gamma_{1} \gamma_{2}} d(t) .
\end{aligned}
$$

The first equation describes how the damping center is driven by 
the external force, whereas the second equation describes how the two masses move with respect to each other.

In case of a constant force $f(t)=1$, with initial conditions $c(0)=0, d(0)=0$ the solutions are:

$$
\begin{aligned}
& c(t)=t, \\
& d(t)=\frac{\alpha \beta\left(\beta \kappa_{1} \gamma_{2}-\alpha \gamma_{1} \kappa_{2}\right)}{\alpha^{2} \gamma_{1}+\beta^{2} \gamma_{2}}\left(1-e^{-\frac{\alpha^{2} \gamma_{1}+\beta^{2} \gamma_{2}}{\alpha \beta \gamma_{1} \gamma_{2}} t}\right) .
\end{aligned}
$$

We can see that the damping center moves at a constant unit velocity, again. The normalized distance $d$ between the masses approaches exponentially a steady state value at the rate of $\left(\alpha^{2} \gamma_{1}+\beta^{2} \gamma_{2}\right) /\left(\alpha \beta \gamma_{1} \gamma_{2}\right)$, this steady state distance is $d^{*}=$ $\alpha \beta\left(\beta \kappa_{1} \gamma_{2}-\alpha \kappa_{2} \gamma_{1}\right) /\left(\alpha^{2} \gamma_{1}+\beta^{2} \gamma_{2}\right)$.

For the dimensionless positions of the masses we obtain

$$
\begin{aligned}
x_{1}(t)= & \frac{\alpha}{\alpha^{2} \gamma_{1}+\beta^{2} \gamma_{2}} t \\
& +\beta \gamma_{2} \frac{\alpha \beta\left(\beta \kappa_{1} \gamma_{2}-\alpha \gamma_{1} \kappa_{2}\right)}{\left(\alpha^{2} \gamma_{1}+\beta^{2} \gamma_{2}\right)^{2}}\left(1-e^{-\frac{\alpha^{2} \gamma_{1}+\beta^{2} \gamma_{2}}{\alpha \beta \gamma_{1} \gamma_{2}} t}\right), \\
x_{2}(t)= & \frac{\beta}{\alpha^{2} \gamma_{1}+\beta^{2} \gamma_{2}} t \\
& -\alpha \gamma_{1} \frac{\alpha \beta\left(\beta \kappa_{1} \gamma_{2}-\alpha \gamma_{1} \kappa_{2}\right)}{\left(\alpha^{2} \gamma_{1}+\beta^{2} \gamma_{2}\right)^{2}}\left(1-e^{-\frac{\alpha^{2} \gamma_{1}+\beta^{2} \gamma_{2}}{\alpha \beta \gamma_{1} \gamma_{2}} t}\right) .
\end{aligned}
$$

The exponential terms disappear with time, and we find in the steady state that the masses move at a constant, but different speed. Note that the coefficients in each term are different, hence with an appropriate choice of the parameters any prescribed final displacement can be reached in any prescribed time. Hence the bodies have different final positions (spatial separation) and they require different time to reach them (temporal separation). Such simultaneous spatial and temporal separation in our overdamped system is very important in understanding the conformational changes of macromolecules. Our model, in essence, is a prototypical mechanical device to investigate the mechanics of conformational changes of, for example, the myosin II motor protein.

\section{Discussion and conclusions}

In biological systems, motor proteins are responsible for many types of motions from cellular transportation through skeletal muscle contraction to peristaltic movements. The number of molecules acting during the motion is in the range of a single molecule (intracellular transportation) and several thousand molecules (macroscopic movements). There are different approaches and models in different disciplines trying to describe the complicated phenomena occurring during motion. In Mechanics, new perspectives were opened by computational methods creating the research field of molecular dynamics, with tentatives of considering all the atoms acting together within a protein. However, despite the increasing capacity of computers, it is not yet possible to model all the atoms of motor proteins, and for the moment, it is hopeless to examine complicated structures with several proteins working together. In the present study, we have found a very simple mechanical device that is capable of separating displacements, and another one, which can separate both displacements and velocities of two bodies. This way, we have found a system, which can produce the same basic operation as a single protein: spatial and temporal separation. Due to the low degree-of-freedom of our model, it can be a convenient building block to model the highly ordered, huge protein structures, like muscle tissue, for example.

The direction of further investigations originates also from biology: under some circumstances, motor proteins seem to "think" between states, which means that they act slower than expected based on the molecular stiffnesses and damping parameters. In our model this could be modelled by inserting a damping element on the excited point. This might lead to more complicated equations, but would not influence the main properties of the model, hence this is a quite straightforward extension of our mechanical device. It is also quite simple to extend our model to include other force functions $F(t)$, that could better model actual energy input into molecular systems.

\section{References}

1 Sztankó K, Active vibration control in rotating shafts, Periodica Polytechnica, Series Mechanical Engineering 49 (2002), 71-78.

2 Takács D, Stépán G, Stability of towed wheels with elastic steering mechanism and shimmy damper, Periodica Polytechnica, Series Mechanical Engineering 51 (2007), 99-103.

3 Barkwell L., Lancaster P., Overdamped and gyroscopic vibrating systems, Journal of Applied Mechanics 59 (1992), 176-181. Overdamped mechanical systems, in particular, gyroscopic systems. Does NOT neglect inertial terms. Engineering staff, they look for the stability of the system.

4 Starek L., Inman D. J., A symmetric inverse vibration problem with overdamped modes, Journal of Sound and Vibration 181 (1995), 893-903.

5 Malinowska J., Sowińska M., Borgieł W., Mechanical properties of human tooth approximated with overdamped oscillators, Acta of Bioengineering and Biomechanics 7 (2005), 59-68.

6 Chopra A. K., Dynamics of Structures: Theory and Applications to Earthquake Engineering, Prentice Hall, 2006.

7 Helgesen G., Pieranski P., Skjeltorp A. T., Nonlinear phenomena in systems of magnetic holes, Physical Review Letters 64 (1990), 1425-1428. Experiments on the behaviour of nonmagnetic spheres in ferrofluid. Inertial effects are negligible due to high viscosity of the ferrofluid and low inertia of particles.

8 Melle S., Fuller G. G., Rubio M. A., Structure and dynamics of magnetorheological fluids in rotating magnetic fields, Physical Review E 61 (2000), 4111-4117. Experiments with motion of colloidal particles in magnetorheological fluids in the presence of external magnetic field. Inertial terms are neglected in their theory. Particles form chains in the fluid.

9 Melle S., Calderón O. G., Rubio M. A., Fuller G. G., Chain rotational dynamics in MR suspensions, International Journal of Modern Physics B 16 (2002), 2293-2299. Colloidal/molecular chains in magnetorheological suspensions subjected to rotating magnetic field: experimenst show that the chain like aggregates rotate synchronously with the magnetic field. Mason number: ratio of viscous to magnetic forces.

10 Dreyfus R., Baudry J., Roper M. L., Fermigier M., Stone H. A., Bibette J., Microscopic artificial swimmers, Nature 437 (2005), 862-865. They attach a linear chain of colloidal magnetic particles, linked by DNA, to red blood cells, and actuate it with an external magnetic field. This system can 
work as artificial flagellum. Inertial forces are very small at the micrometer scale as compared with the viscous forces.

11 Qian B., Powers T. R., Breuer K. S., Shape transition and propulsive force of an elastic rod rotating in a viscous fluid, Physical Review Letters 100 (2008), no. 078101. They model flagella and cilia by rotated rod. They consider the fluid-structure interaction in a viscous fluid, where, at these micron scales, the viscous effects dominate inertia.

12 Qian M., Zhu S., Qin W.-X., Dynamics in a chain of overdamped pendula driven by constant torques, SIAM Journal of Applied Mathematics 57 (1997), 294-305. Coupled oscillators, they model Josephson junction arrays or oscillating chemical reactions or neural networks. Now: overdamped oscillators with nearest neighbour coupling. Inertial terms are neglected. They find either limit cycles or fixed points.

13 De Luca R., Strongly coupled overdamped pendulums, Revista Brasileira de Ensino de Física 30 (2008), no. 4304. Coupled oscillators, inertial terms are neglected. The system is similar to Josephson junctions. Print version ISSN 1806-1117.

14 Gammaitoni L., Hänggi P., Jung P., Marchesoni F., Stochastic resonance, Reviews of Modern Physics 70 (1998), 223-287. They use, as a generic model, the overdamped motion of a Brownian particle in a bistable potential in the presence of noise and periodic forcing to investigate stochastic resonance.

15 Evstigneev M., Reimann P., Pankov V., Prince R.H., Stochastic resonance in monostable overdamped systems, Europhysics Letters 65 (2004), 7-12. Noise driven nonlinear oscillator with harmonic forcing: stochastic resonance occurs despite the fact that the system in monostable. System: Brownian particle. Inertial effects are neglected.

16 Shelton W. A., Bonin K. D., Walker T. G., Nonlinear motion of optically torqued nanorods, Physical Review E 71 (2005), no. 036204. Experiments with glass nanorods, held by optical tweezers, in water. Inertial effects are neglected, they give estimate on the order of the effects of the various terms in the dimensionless equation of motion.

17 Bender C. M., Orszag S. A., Advanced mathematical methods for scientists and engineers: asymptotic methods and perturbation theory, Springer, 1999.

18 Bagshaw C. R., Muscle contraction, Kluwer Academic Publishers, 1993.

19 Howard J, Mechanics of motor proteins and thy cytoskeleton, Sinauer Associates, 2001. 